\title{
Online Survey on Accessing Psychological Knowledge and Interventions During the COVID-19 Pandemic - China, 2020
}

\author{
Qingdong $\mathrm{Lu}^{1,2}$; Lin $\mathrm{Liu}^{1,2}$; Yunhe Wang ${ }^{1,2}$; Le Shi ${ }^{3}$; Yingying $\mathrm{Xu}^{1,2}$; Zhengan $\mathrm{Lu}^{3}$; Jianyu Que \\ Jingli Yue ${ }^{3}$; Kai Yuan"; Wei Yan ${ }^{3}$; Yankun Sun ${ }^{3}$; Jie Shi ${ }^{1, * *}$; Yanping Bao ${ }^{1,2, *}$; Lin $\mathrm{Lu}^{3,4, *}$
}

\section{Summary \\ What is already known on this topic? \\ The public was at elevated risk of mental health illnesses during the coronavirus disease 2019 (COVID- 19) pandemic, so accessibility to psychological knowledge and interventions is vital to promptly respond to mental health crises. \\ What is added by this report? \\ During the pandemic period, 40,724 (71.9\%) participants reportedly had access to psychological knowledge, and 36,546 (64.5\%) participants had accessed information on psychological interventions. Participants who were male, unmarried, living alone, divorced or widowed, or infected with COVID-19 were less likely to access psychological knowledge and intervention. \\ What are the implications for public health practice? \\ Governments should pay more attention to formulate policies, popularize psychological education, and provide mental health services online or in the community.}

The public was at elevated risk of mental health problems during the coronavirus disease 2019 (COVID-19) pandemic. In this article, we investigated the pattern and risk factors of accessing psychological information during the pandemic in China. From February 28 to March 11, 2020, a cross-sectional online survey was conducted in all provincial-level administrative divisions (PLADs) of China to obtain general demographic characteristics, access history for psychological knowledge and interventions, psychological status, and other information. Multivariable logistic regression was used to explore the influencing factors of accessing psychological information and intervention among the general population. A total of 56,679 people were included in the survey. During the pandemic period, 40,724 (71.9\%) reported had access to psychological knowledge, and 36,546 (64.5\%) people accessed information on psychological intervention. Governments and professionals need to pay more attention to increase the accessibility of mental health resources during the pandemic in the future.

COVID-19 has spread across the world and galvanized global action since its emergence in late December 2019 (1). The unpredictability and uncertainty of the COVID-19 pandemic, associated containment strategies, and financial losses are among the major stressors that undoubtedly contributed to widespread emotional distress and increased the risk for psychiatric illness associated with COVID-19 (2). The prevalence of depression, anxiety, insomnia, and other mental health problems was high among COVID-19 patients, healthcare workers, and the general population (3-4). The World Health Organization (WHO) Director-General estimated that the COVID19 pandemic had impacted the mental health of millions of people, yet relatively few people have access to quality mental health services (5). The high burden of mental health problems calls for earlier psychological interventions and timely mental health responses during the COVID-19 pandemic (2).

China has started to address the increasing need for mental health services as the Healthy China Action 2030 plan implemented a series of programs including improving mental health literacy of residents $(\sigma)$. After the emergence of COVID-19, a series of mental health measures were undertaken to address the demands of COVID-19 mental health concerns by adapting mental health services from China, other national governments, and international organizations $(2,7-8)$. This study assessed the demographics and access histories of psychological knowledge and interventions to provide evidence for policymakers and popularize psychological health resources for China's public in the COVID-19 era.

This cross-sectional online survey was conducted from February 28, 2020 to March 11, 2020. A questionnaire was designed during the pandemic and 
delivered through an online e-health platform Joybuy, which is an e-commerce platform launched by Jingdong for the trading of medicine-related products as detailed in our previous study (3). Written informed consent was received online before the respondents began the questionnaire. A total of 56,932 participants provided informed consent and submitted the questionnaires, and after the quality control by age, 56,679 participants from all PLADs in China were included.

The access histories of psychological knowledge and interventions were measured by the following question: "Did you access the information about psychological knowledge/psychological interventions before or during the COVID-19 pandemic through the media (TV, mobile phone, internet, newspaper, etc.)?" Psychological knowledge refers to causes, diagnoses, symptoms, preventive measures, and basic coping strategies to alleviate the psychological effects of common mental disorders such as depression, anxiety, insomnia, and post-traumatic stress disorder. The timing "before the pandemic" referred to daily life before the pandemic, and "during the pandemic" referred to the period between the national state of emergency being declared and the time answering the questionnaire. Self-reported difficulties in accessing the information before and during the pandemic were measured using the visual analogue scale (VAS), which ranged from 0 (no difficulty at all) to 10 (very difficult).

To explore factors potentially associated with accessing psychological knowledge and interventions, univariate logistic regression was performed followed by multivariable logistic regression analysis to calculate the odds ratios (ORs) and 95\% confidence intervals (95\% CI) of possible confounders and adjusting for potential covariates. The statistical analyses were performed using SPSS statistical software (IBM Corporation, version 25.0).

The mean \pm SD age of the participants was $36.0 \pm 8.2$ years, 27,149 (47.9\%) of them were male, 44,274 $(78.1 \%)$ were married or cohabiting. Additional demographic and characteristic information was presented in Table 1.

Overall, 40,724 (71.9\%) of the participants accessed psychological knowledge during the COVID-19 pandemic, which was higher than the 33,553 (59.2\%) before the pandemic, and 36,546 (64.5\%) participants accessed information on psychological interventions, which was also higher than the $27,043(47.7 \%)$ before the pandemic (Table 2). Individuals with and without mental health symptoms had higher rates of accessing both psychological knowledge and interventions during the pandemic than before the pandemic.

Multivariable logistic regression analyses showed that the participants who were male $(\mathrm{OR}=1.42,95 \%$ CI: 1.36-1.49), unmarried $(\mathrm{OR}=1.16,95 \% \mathrm{CI}$ : 1.10-1.24), lived alone, divorced, or widowed $(\mathrm{OR}=1.22$, 95\% CI: $1.06-1.41)$, or infected with COVID-19 (OR=2.35, 95\% CI: 1.27-4.34) had less chance to access psychological knowledge. Meanwhile, people who were older (aged $41-50$ years: $\mathrm{OR}=0.80$, 95\% CI: $0.74-0.86$; $>50$ years: $\mathrm{OR}=0.68,95 \% \mathrm{CI}$ : 0.60-0.77; reference group: $18-30$ years), were frontline workers $(\mathrm{OR}=0.85,95 \% \mathrm{CI}: 0.80-0.91)$, had a family members served as frontline workers $(\mathrm{OR}=0.81, \quad 95 \%$ CI: 0.77-0.85), experienced quarantine $(\mathrm{OR}=0.83,95 \% \mathrm{CI}: 0.79-0.87)$, traffic restrictions $\quad(\mathrm{OR}=0.78, \quad 95 \% \quad \mathrm{CI}: \quad 0.73-0.82)$, community containment $\quad(\mathrm{OR}=0.85, \quad 95 \% \quad \mathrm{CI}$ : $0.78-0.93)$, have mental health symptoms $(\mathrm{OR}=0.78$, 95\% CI: $0.75-0.82)$ and accessed psychological knowledge $(\mathrm{OR}=0.30,95 \% \mathrm{CI}: 0.28-0.31)$ and interventions (OR=0.22, 95\% CI: $0.20-0.23$ ) before the pandemic were more likely to access psychological knowledge during the pandemic.

Similarly, the factors that deteriorated the odds of obtaining information on psychological interventions were being male $(\mathrm{OR}=1.44,95 \% \mathrm{CI}: 1.38-1.50)$, unmarried $(\mathrm{OR}=1.11,95 \%$ CI: 1.05-1.18), living alone, divorced, or widowed $(\mathrm{OR}=1.22,95 \% \mathrm{CI}$ : 1.06-1.40), and infected with COVID-19 (OR=1.86, 95\% CI: $1.00-3.45)$. The protective factors were being older (aged 41-50: OR=0.82, 95\% CI: 0.76-0.88; $>50$ : $\mathrm{OR}=0.71,95 \% \mathrm{CI}: 0.64-0.80)$, living in the countryside (OR=0.86, 95\% CI: 0.78-0.94), being frontline workers $(\mathrm{OR}=0.79$, 95\% CI: 0.74-0.84), having a family members or friends that were frontline workers $(\mathrm{OR}=0.81,95 \% \mathrm{CI}: 0.77-0.85)$, experiencing quarantine $(\mathrm{OR}=0.86,95 \% \mathrm{CI}: 0.82-0.90)$, traffic restrictions $\quad(\mathrm{OR}=0.76, \quad 95 \% \quad \mathrm{CI}: \quad 0.72-0.80)$, community containment $\quad(\mathrm{OR}=0.80, \quad 95 \% \quad \mathrm{CI}$ : 0.74-0.88), having symptoms of mental health disorders $\quad(\mathrm{OR}=0.72,95 \% \quad \mathrm{CI}: \quad 0.69-0.76)$, and accessing psychological knowledge $(\mathrm{OR}=0.57,95 \% \mathrm{CI}$ : $0.54-0.60)$ and interventions $(\mathrm{OR}=0.12,95 \% \mathrm{CI}$ : $0.12-0.12$ ) before the pandemic (Table 3 ).

\section{DISCUSSION}

This nationwide survey showed that about $71.9 \%$ 
TABLE 1. Demographic characteristics and epidemic-related information for the sample during the COVID-19 pandemic China, 2020.

\begin{tabular}{|c|c|c|c|}
\hline Factors & Total, N (\%) & $\begin{array}{c}\text { No. of people accessing } \\
\text { psychological knowledge }(\%)\end{array}$ & $\begin{array}{c}\text { No. of people accessing } \\
\text { information of interventions (\%) }\end{array}$ \\
\hline Overall & 56,679 & $40,724(71.9)$ & $36,546(64.5)$ \\
\hline \multicolumn{4}{|l|}{ Age, years } \\
\hline $18-30$ & $16,142(28.5)$ & $11,548(71.5)$ & $10,537(65.3)$ \\
\hline $31-40$ & $26,824(47.3)$ & $18,978(70.8)$ & $16,890(63.0)$ \\
\hline $41-50$ & $11,215(19.8)$ & $8,283(73.9)$ & $7,406(66.0)$ \\
\hline$>50$ & $2,498(4.4)$ & $1,915(76.7)$ & $1,713(68.6)$ \\
\hline \multicolumn{4}{|l|}{ Gender } \\
\hline Female & $29,530(52.1)$ & $21,847(74.0)$ & $19,767(66.9)$ \\
\hline Male & $27,149(47.9)$ & $18,877(69.5)$ & $16,779(61.8)$ \\
\hline \multicolumn{4}{|l|}{ Area type } \\
\hline Urban & $52,839(93.2)$ & $37,862(71.7)$ & $33,896(64.1)$ \\
\hline Rural & $3,840(6.8)$ & $2,862(74.5)$ & $2,650(69.0)$ \\
\hline \multicolumn{4}{|l|}{ Level of education } \\
\hline Less than senior school & $2,084(3.7)$ & $1,578(75.7)$ & $1,448(69.5)$ \\
\hline Senior school & $7,456(13.2)$ & $5,780(77.5)$ & $5,227(70.1)$ \\
\hline College degree or higher & $47,139(83.2)$ & $33,366(70.8)$ & $29,871(63.4)$ \\
\hline \multicolumn{4}{|l|}{ Marital status } \\
\hline Married or cohabiting & $44,274(78.1)$ & $32,213(72.8)$ & $28,870(65.2)$ \\
\hline Unmarried & $11,135(19.6)$ & $7,626(68.5)$ & $6,892(61.9)$ \\
\hline Live apart, divorced, or widowed & $1,270(2.2)$ & $885(69.7)$ & $784(61.7)$ \\
\hline \multicolumn{4}{|l|}{ Monthly family income, RMB } \\
\hline$\leq 5,000$ & $13,016(23.0)$ & $9,665(74.3)$ & $8,771(67.4)$ \\
\hline $5,000-7,999$ & $13,663(24.1)$ & $10,050(73.6)$ & $9,056(66.3)$ \\
\hline $8,000-11,999$ & $12,829(22.6)$ & $9,270(72.3)$ & $8,240(64.2)$ \\
\hline$\geq 12,000$ & $17,171(30.3)$ & $11,739(68.4)$ & $10,479(61.0)$ \\
\hline \multicolumn{4}{|l|}{ History of chronic diseases } \\
\hline Yes & $3,274(5.8)$ & $2,359(72.1)$ & $2,068(63.2)$ \\
\hline No or unknown & $53,405(94.2)$ & $38,365(71.8)$ & $34,478(64.6)$ \\
\hline \multicolumn{4}{|l|}{ History of mental disorders } \\
\hline Yes & $161(0.3)$ & $109(67.7)$ & $103(64.0)$ \\
\hline No or unknown & $56,518(99.7)$ & $40,615(71.9)$ & $36,443(64.5)$ \\
\hline \multicolumn{4}{|l|}{ Family history of mental disorders } \\
\hline Yes & $396(0.7)$ & $272(68.7)$ & $238(60.1)$ \\
\hline No or unknown & $56,283(99.3)$ & $40,452(71.9)$ & $36,308(64.5)$ \\
\hline \multicolumn{4}{|l|}{ Sleep disturbance } \\
\hline Yes & $15,981(28.2)$ & $11,756(73.6)$ & $10,642(66.6)$ \\
\hline No & $40,698(71.8)$ & $28,968(71.2)$ & $25,904(63.6)$ \\
\hline \multicolumn{4}{|l|}{ Smoking } \\
\hline Yes & $6,965(12.3)$ & $5,111(73.4)$ & $4,543(65.2)$ \\
\hline No & $49,714(87.7)$ & $35,613(71.6)$ & $32,003(64.4)$ \\
\hline
\end{tabular}


TABLE 1. (Continued)

\begin{tabular}{|c|c|c|c|}
\hline Factors & Total, N (\%) & $\begin{array}{c}\text { No. of people accessing } \\
\text { psychological knowledge (\%) }\end{array}$ & $\begin{array}{c}\text { No. of people accessing } \\
\text { information of interventions (\%) }\end{array}$ \\
\hline \multicolumn{4}{|l|}{ Alcohol consumption } \\
\hline Yes & $5,145(9.1)$ & $3,789(73.6)$ & $3,423(66.5)$ \\
\hline No & $51,534(90.9)$ & $36,935(71.7)$ & $33,123(64.3)$ \\
\hline \multicolumn{4}{|l|}{ Infection status of COVID-19 } \\
\hline Confirmed or suspected & $100(0.2)$ & $60(60.0)$ & $59(59.0)$ \\
\hline Uninfected & $56,579(99.8)$ & $40,664(71.9)$ & $36,487(64.5)$ \\
\hline \multicolumn{4}{|c|}{ Have infected family members } \\
\hline Yes & $608(1.1)$ & $434(71.4)$ & $397(65.3)$ \\
\hline No & $56,071(98.9)$ & $40,290(71.9)$ & $36,149(64.5)$ \\
\hline \multicolumn{4}{|c|}{ Close contact with patients infected with COVID-19 } \\
\hline Yes & $219(0.4)$ & $157(71.7)$ & $143(65.3)$ \\
\hline No & $56,460(99.6)$ & $40,567(71.9)$ & $36,403(64.5)$ \\
\hline \multicolumn{4}{|c|}{ Live in Hubei Province during pandemic } \\
\hline Yes & $2,352(4.1)$ & $1,662(70.7)$ & $1,522(64.7)$ \\
\hline No & $54,327(95.9)$ & $39,062(71.9)$ & $35,024(64.5)$ \\
\hline \multicolumn{4}{|c|}{ Have you been to Hubei Province before the outbreak } \\
\hline Yes & $2,452(4.3)$ & $1,727(70.4)$ & $1,585(64.6)$ \\
\hline No & $54,227(95.7)$ & $38,997(71.9)$ & $34,961(64.5)$ \\
\hline \multicolumn{4}{|c|}{ Participation of frontline work related to the outbreak } \\
\hline Yes & $9,725(17.2)$ & $7,680(79.0)$ & $7,111(73.1)$ \\
\hline No & $46,954(82.8)$ & $33,044(70.4)$ & $29,435(62.7)$ \\
\hline \multicolumn{4}{|c|}{ Family members were frontline workers } \\
\hline Yes & $17,587(31.0)$ & $13,646(77.6)$ & $12,475(70.9)$ \\
\hline No & $39,092(69.0)$ & $27,078(69.3)$ & $24,071(61.6)$ \\
\hline \multicolumn{4}{|c|}{ Experience of traffic restriction } \\
\hline Yes & $44,762(79.0)$ & $33,002(73.7)$ & $29,877(66.7)$ \\
\hline No & $11,917(21.0)$ & $7,722(64.8)$ & $6,669(56.0)$ \\
\hline \multicolumn{4}{|c|}{ Experience of community containment } \\
\hline Yes & $53,076(93.6)$ & $38,444(72.4)$ & $34,578(65.1)$ \\
\hline No & $3,603(6.4)$ & $2,280(63.3)$ & $1,968(54.6)$ \\
\hline \multicolumn{4}{|l|}{ Experience of quarantine } \\
\hline Centralized or at home & $16,454(29.0)$ & $12,555(76.3)$ & $11,389(69.2)$ \\
\hline No & $40,225(71.0)$ & $28,169(70.0)$ & $25,157(62.5)$ \\
\hline \multicolumn{4}{|c|}{ Method of work during the pandemic } \\
\hline Work at home & $7,427(13.1)$ & $5,330(71.8)$ & $4,794(64.5)$ \\
\hline Work at institution & $29,498(52.0)$ & $21,009(71.2)$ & $18,718(64.5)$ \\
\hline Not back to work & $19,754(34.9)$ & $14,385(72.8)$ & $13,034(66.0)$ \\
\hline \multicolumn{4}{|c|}{ Mental health symptoms (depression, anxiety, insomnia, or acute distress) } \\
\hline Any symptoms & $26,680(47.1)$ & $20,059(75.2)$ & $18,402(69.0)$ \\
\hline No & $29,999(52.9)$ & 20,665 (68.9) & $18,144(60.5)$ \\
\hline
\end{tabular}


TABLE 2. Access history and difficulty accessing psychological knowledge and interventions during the COVID-19 pandemic - China, 2020.

\begin{tabular}{lcc}
\hline Factors & Before COVID-19 pandemic & During COVID-19 pandemic \\
\hline Access to psychological knowledge $(\mathrm{N}, \%)$ & $33,553(59.2)$ & $40,724(71.9)$ \\
Total & $16,381(61.4)$ & $20,059(75.2)$ \\
People with mental health symptoms & $17,172(57.2)$ & $20,665(68.9)$ \\
People without mental health symptoms & & $36,546(64.5)$ \\
Access to psychological intervention information (N, \%) & $27,043(47.7)$ & $18,402(69.0)$ \\
Total & $13,708(51.4)$ & $18,144(60.5)$ \\
People with mental health symptoms & $13,335(44.5)$ & $3.26 \pm 2.52^{*}$ \\
People without mental health symptoms & $3.31 \pm 2.61$ & $4.07 \pm 2.43^{*}$ \\
Difficulty in getting access to psychological intervention information (mean $\pm \mathrm{SD})$ & $2.55 \pm 2.38^{*}$ \\
Total & $4.01 \pm 2.53$ & $2.69 \pm 2.53$ \\
People with mental health symptoms & & \\
People without mental health symptoms & & \\
\hline
\end{tabular}

* The difference between before and during the pandemic was statistically significant $(p<0.05)$.

and $64.5 \%$ of participants had accessed psychological knowledge and interventions during the pandemic. This study identified the associated factors hindering the accessibility of psychological information and intervention during the COVID-19 pandemic among subpopulations, which provided information to improve the availability of mental health services and addressing the mental health wellbeing during the pandemic. Accordingly, the Chinese government has prioritized mental health services since the beginning of the pandemic ( 7 ), and the National Health Commission of China has published several guiding documents and guidelines for emergency psychological crisis interventions for the COVID-19 epidemic (9).

Participants infected with COVID-19 reported less accessibility to information and interventions, and confirmed patients may focus on their own physical condition so they neglect their mental health. The Chinese government implemented programs to improve access to psychological counseling, social work services, and comprehensive psychological rehabilitation for COVID-19 patients, isolation personnel, and their families in the post-pandemic period (10). Moreover, more choices for psychological treatment and interventions should be provided for individuals with mental health problems, especially the integration of more online and field psychological resources in the future.

The findings in this study were subject to at least three limitations. First, this was an online survey, and a convenience sampling method was used. Although this study had extensive geographic coverage across China and a large sample size, it was conducted among internet users who were young and highly educated; thus, the representativeness of the sample might be limited. Second, people's accessibility information comes from a single self-reported question and there were no repeated verification problems and detailed resources included in this questionnaire, so the accessibility of psychological resources should be addressed in the future. Third, because this was a crosssectional study, the associations could only be established at the population level. Future studies, especially large cohort studies of the population, are needed.

Mental health literacy is a target of the Healthy China Action and Healthy China 2030 Plan and a call to action for the general population. Governments, researchers, and educators should pay more attention to formulating policies, popularizing psychological education, and providing mental health services online and in the community.

Acknowledgments: We appreciate the contributions of Jingdong.

Conflicts of interest: No conflicts of interest were reported.

Fundings: This study was supported by grant BMU2020HKYZX008 from the Special Research Fund of PKUHSC for Prevention and Control of COVID-19 and the Fundamental Research Funds for the Central Universities, grant 2019YFA0706200 and 2020YFC2003600 from the National Key Research and Development Program of China, and grant 81761128036 from the National Natural Science Foundation of China-Canadian Institutes of Health Research Joint Health Research Program. 
TABLE 3. Multivariable logistic regressions of risk factors associated with accessing knowledge and intervention during the COVID-19 pandemic - China, 2020.

\begin{tabular}{|c|c|c|c|c|}
\hline \multirow{2}{*}{ Variables } & \multicolumn{2}{|c|}{$\begin{array}{c}\text { Risk of not accessing psychological } \\
\text { knowledge }\end{array}$} & \multicolumn{2}{|c|}{$\begin{array}{l}\text { Risk of not accessing psychologica } \\
\text { interventions }\end{array}$} \\
\hline & $\mathrm{aOR}^{*}(95 \% \mathrm{Cl})$ & $p$-value & $\mathrm{aOR}^{*}(95 \% \mathrm{Cl})$ & $p$-value \\
\hline \multicolumn{5}{|l|}{ Age, years } \\
\hline $18-30$ & 1 (Reference) & & 1 (Reference) & \\
\hline $31-40$ & $0.97(0.92-1.03)$ & 0.280 & $1.00(0.95-1.06)$ & 0.952 \\
\hline $41-50$ & $0.80(0.74-0.86)$ & $<0.001$ & $0.82(0.76-0.88)$ & $<0.001$ \\
\hline$>50$ & $0.68(0.60-0.77)$ & $<0.001$ & $0.71(0.64-0.80)$ & $<0.001$ \\
\hline \multicolumn{5}{|l|}{ Gender } \\
\hline Female & 1 (Reference) & & 1 (Reference) & \\
\hline Male & $1.42(1.36-1.49)$ & $<0.001$ & $1.44(1.38-1.50)$ & $<0.001$ \\
\hline \multicolumn{5}{|l|}{ Areas types } \\
\hline Urban & - & & 1 (Reference) & \\
\hline Rural & - & - & $0.86(0.78-0.94)$ & 0.001 \\
\hline \multicolumn{5}{|l|}{ Marital status } \\
\hline Married or cohabiting & 1 (Reference) & & 1 (Reference) & \\
\hline Unmarried & $1.16(1.10-1.24)$ & $<0.001$ & $1.11(1.05-1.18)$ & $<0.001$ \\
\hline Live alone, divorced, or widowed & $1.22(1.06-1.41)$ & 0.006 & $1.22(1.06-1.40)$ & 0.006 \\
\hline \multicolumn{5}{|l|}{ Infection status of COVID-19 } \\
\hline Uninfected & 1 (Reference) & & 1 (Reference) & \\
\hline Confirmed or suspected & $2.35(1.27-4.34)$ & 0.006 & $1.86(1.00-3.45)$ & 0.049 \\
\hline \multicolumn{5}{|c|}{ Participation of frontline work related to the outbreak } \\
\hline No & 1 (Reference) & & 1 (Reference) & \\
\hline Yes & $0.85(0.80-0.91)$ & $<0.001$ & $0.79(0.74-0.84)$ & $<0.001$ \\
\hline \multicolumn{5}{|l|}{ Family members were frontline workers } \\
\hline No & 1 (Reference) & & 1 (Reference) & \\
\hline Yes & $0.81(0.77-0.85)$ & $<0.001$ & $0.81(0.77-0.85)$ & $<0.001$ \\
\hline \multicolumn{5}{|l|}{ Experienced traffic restriction } \\
\hline No & 1 (Reference) & & 1 (Reference) & \\
\hline Yes & $0.78(0.73-0.82)$ & $<0.001$ & $0.76(0.72-0.80)$ & $<0.001$ \\
\hline \multicolumn{5}{|l|}{ Experienced community containment } \\
\hline No & 1 (Reference) & & 1 (Reference) & \\
\hline Yes & $0.85(0.78-0.93)$ & 0.001 & $0.80(0.74-0.88)$ & $<0.001$ \\
\hline \multicolumn{5}{|l|}{ Experienced quarantine } \\
\hline No & 1 (Reference) & & 1 (Reference) & \\
\hline Centralized or at home & $0.83(0.79-0.87)$ & $<0.001$ & $0.86(0.82-0.90)$ & $<0.001$ \\
\hline \multicolumn{5}{|l|}{ Mental health symptoms } \\
\hline No & 1 (Reference) & & 1 (Reference) & \\
\hline Yes & $0.78(0.75-0.82)$ & $<0.001$ & $0.72(0.69-0.76)$ & $<0.001$ \\
\hline \multicolumn{5}{|c|}{ Access to psychological knowledge before pandemic } \\
\hline Yes & 1 (Reference) & & 1 (Reference) & \\
\hline No & $0.30(0.28-0.31)$ & $<0.001$ & $0.57(0.54-0.60)$ & $<0.001$ \\
\hline \multicolumn{5}{|c|}{ Access to psychological interventions before pandemic } \\
\hline Yes & 1 (Reference) & & 1 (Reference) & \\
\hline No & $0.22(0.20-0.23)$ & $<0.001$ & $0.12(0.12-0.12)$ & $<0.001$ \\
\hline
\end{tabular}


doi: $10.46234 / \mathrm{ccdcw} 2020.218$

\# Corresponding authors: Jie Shi, shijie@bjmu.edu.cn; Yanping Bao, baoyp@bjmu.edu.cn; Lin Lu, linlu@bjmu.edu.cn.

\footnotetext{
${ }^{1}$ National Institute on Drug Dependence and Beijing Key Laboratory of Drug Dependence, Peking University, Beijing, China; 2 School of Public Health, Peking University, Beijing, China; ${ }^{3}$ Institute of Mental Health, National Clinical Research Center for Mental Disorders, Key Laboratory of Mental Health and Peking University Sixth Hospital, Peking University, Beijing, China;

${ }^{4}$ Peking-Tsinghua Center for Life Sciences and PKU-IDG/McGovern Institute for Brain Research, Peking University, Beijing, China.
}

Submitted: September 18, 2020; Accepted: September 30, 2020

\section{REFERENCES}

1. Wang C, Horby PW, Hayden FG, Gao GF. A novel coronavirus outbreak of global health concern. Lancet 2020;395(10223):470 - 73 http://dx.doi.org/10.1016/S0140-6736(20)30185-9.

2. Moreno C, Wykes T, Galderisi S, Nordentoft M, Crossley N, Jones N, et al. How mental health care should change as a consequence of the COVID-19 pandemic. Lancet Psychiatry 2020;7(9):813-24. http://dx.doi.org/10.1016/S2215-0366(20)30307-2.

3. Shi L, Lu ZA, Que JY, Huang XL, Liu L, Ran MS, et al. Prevalence of and risk factors associated with mental health symptoms among the general population in china during the coronavirus disease 2019 pandemic. JAMA Netw Open 2020;3(7):e2014053. http://dx.doi.org/10. 1001/jamanetworkopen.2020.14053.

4. Que JY, Shi L, Deng JH, Liu JJ, Zhang L, Wu SY, et al. Psychological impact of the COVID-19 pandemic on healthcare workers: a crosssectional study in China. Gen Psychiatr 2020;33(3):e100259. http://dx.doi.org/10.1136/gpsych-2020-100259.

5. Ghebreyesus TA. COVID-19 pandemic affects mental health of millions of people: WHO chief. Aug 28, 2020. http://www. xinhuanet.com/english/2020-08/28/c_139323101.htm. [2020-08-28].

6. Bao YP, Meng SQ, Sun YK, Jie S, Lu L. Healthy China Action plan empowers child and adolescent health and wellbeing. Lancet Public Health 2019;4(9):e448. http://dx.doi.org/10.1016/S2468-2667(19) 30164-1.

7. Bao YP, Sun YK, Meng SQ, Shi J, Lu L. 2019-nCoV epidemic: address mental health care to empower society. Lancet 2020;395(10224):e37 8. http://dx.doi.org/10.1016/S0140-6736(20)30309-3.

8. Xiang YT, Zhao YJ, Liu ZH, Li XH, Zhao N, Cheung T, et al. The COVID-19 outbreak and psychiatric hospitals in China: managing challenges through mental health service reform. Int J Biol Sci 2020; 16(10):1741 - 44. http://dx.doi.org/10.7150/ijbs. 45072.

9. Liu S, Yang LL, Zhang CX, Xiang YT, Liu ZC, Hu SH, et al. Online mental health services in China during the COVID-19 outbreak. Lancet Psychiatry 2020;7(4):e17 - 8. http://dx.doi.org/10.1016/S22150366(20)30077-8.

10. State Council Joint Prevention and Control Team to Response to New Coronavirus Pneumonia. Notice on Psychological Counseling and Social Work Service Program for COVID-19 Patients, Quarantined Persons and Their Families. http://www.nhc.gov.cn/jkj/s5888/202004/ 32701ef2e5c940cb8e0e416dbabb4a4d.shtml. [2020-04-07]. (In Chinese). 